\title{
Kalkulieren, restrukturieren, optimieren, (sich) perfektionieren
}

\author{
Der Dienstleistungsbereich Gesundheit besitzt vor dem Hintergrund der Vier- \\ Generationen-Gesellschaft, innovativer Versorgungsformen, Ausgabendämp- \\ fungen und doch anhaltend hoher Steigerungsraten bei Gesundheitsaus- \\ gaben eine hohe Bedeutung.
}

Peter K. Link*

\begin{abstract}
* Der Autor ist Bildungsexperte und Unternehmerberater. Als studierter Wirtschaftswissenschaftler hält er einen MBA in «Management Consultancy» (with distinction) der britischen University of Lincoln, Kingstonupon-Hull, und ein Diplom in Betriebswirtschaft der deutschen Hochschule für Wirtschaft, Ludwigshafen. Nach mehreren Assignments in international arbeitenden Hochschulen ist er heute als Head of Postgraduate Studies an der Privaten Hochschule Wirtschaft Bern tätig.
\end{abstract}

Résumé voir page suivante

\section{Korrespondenz:}

Peter K. Link

Head of Postgraduate Studies Private Hochschule Wirtschaft Parkterrasse 14/16

CH-3012 Bern

Tel. 0313100816

Fax 0313100811

peter.link@feusi.ch

www.phw.bfh.ch
Ärztinnen und Ärzte sind dem Wandel im «Health Sector» in besonders exponierter Position ausgesetzt. Die erworbenen medizinischen Kompetenzen sowie anschliessende Spezialisierungen reichen oft nicht mehr aus. Ein Paradigmenwechsel in der Gesundheitsbranche ist vollzogen. Diese Erkenntnis ist weder neu noch überraschend. Erstaunlich wäre eher die fehlende oder mangelnde Bereitschaft, das veränderte berufliche Selbstverständnis anzuerkennen und neue Know-how-Felder in die Wissenslandkarte ärztlicher Kompetenz mit aufzunehmen - das Management von Einheiten, Abteilungen, Institutionen und Netzwerken im Gesundheitswesen auf allen Ebenen und in jeder Funktion.

Für einen Vertreter der Wirtschaft mag es kaum vorstellbar sein, nach Eindrücken deutscher Krankenhausmanager und Unternehmensberater ist es Realität: Der Chef einer grossen medizinischen Abteilung weiss in der Regel weder, was seine Leistung kostet, noch was sie seiner Organisation einbringt. Er kann nicht beziffern, was die Energie für 35 Minuten Operationszeit kostet, er weiss nicht, wie teuer das OP-Team für eine komplizierte Hüftgelenkendoprothese ist, und er hat keine Ahnung, auf welche Summe sich ein Röntgenbild summiert, das mittels moderner Geräte erstellt, in die entsprechende Abteilung getragen, gelesen und vom Arzt interpretiert werden muss. Wie soll er planen? Wo

\section{Managementkenntnisse} und betriebswirtschaftliches

Know-how stellen eine notwendige Bedingung für das berufliche Überleben dar. optimieren? Wie kann er den Aufwand pro Patient steuern, Budgets kalkulieren oder Kosten senken, um im Wettbewerb mit anderen Häusern bestehen zu können?

\section{Die Survival Skills 2006-2015 für Mediziner im Schnellüberblick}

\section{Finance \& Controlling}

«Wer seine Kosten nicht im Griff hat, macht keine Gewinne». Wie steht es um meine Liquidität, meine Rentabilität, meine Sicherheit und meinen Kapitalumschlag? Die systematische Erhebung finanzieller Kennzahlen zum Zweck einer klar ausgerichteten Unternehmenssteuerung ist als erfolgskritisch zu sehen.

\section{Strategische Führung}

Strategische Orientierungen oder Stossrichtungen sind der allgemeine Kurs, die Generallinie einer Unternehmung. Strategien sind langfristige Grundsatzentscheidungen mit Instrumentalcharakter, ein langfristiger, grundsätzlicher Rahmenplan zur Erreichung der definierten Ziele. Ist die erwünschte Entwicklung, beispielsweise ungleich der erwarteten Entwicklung entsteht eine Ziellücke. Eine Unternehmung gleich welcher Art und Ausrichtung - muss sich neuer Strategien bedienen, um eben die Lücke zu schliessen. Als übergeordnetes Ziel unternehmerischer Aktivität kann ruhig die Wertsteigerung gesehen werden, aber auch die Discounted-FreeCashflow-Methode als ökonomisch fundierte Berechnungsweise des Unternehmenswertes. «Strategie ist die Kunst, sich zu kratzen, bevor es einen juckt.» (Prof. Dr. Kurt Nagel)

\section{Informationstechnologien}

Was hat IT mit BWL zu tun? IT-Projekte haben immer etwas von «Murphy's Law»: Was schief 


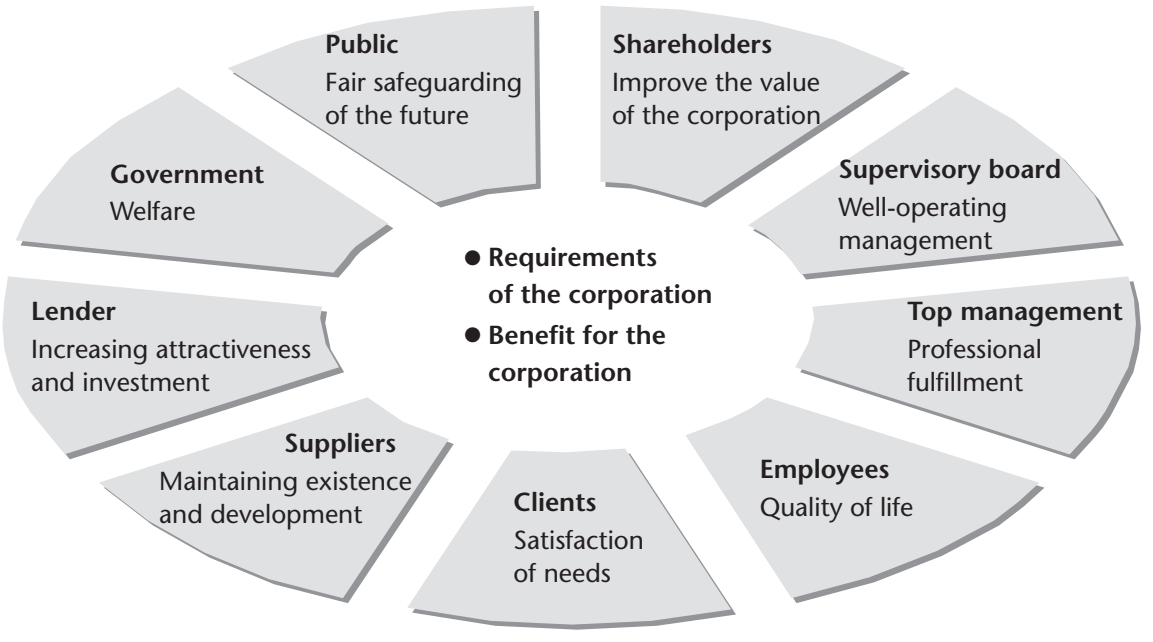

gehen kann, geht schief. Zeitpläne werden überschritten, Kosten laufen aus dem Ruder, unzählige Pleiten und Pannen begleiten den Prozess. Positivbeispiel: Mehrere Regionalspitäler schaffen eine neue Softwaregeneration an. Im Rahmen eines Workshops wird schnell klar, dass die Informationstechnologie der unterschiedlichen Spitäler weitestgehend deckungsgleich ist. Die anschliessende und gemeinsame Konzentration auf das Projektmanagement und Controlling verschafft dem Verbund die Software zu einem deutlich günstigeren Preis. «Ein Gramm Information wiegt schwerer als tausend Tonnen Meinung.» (Gerd Bacher)

\section{Qualitätsmanagement}

Der Mensch mit seinen Fähigkeiten und Bedürfnissen als der zentrale Teil in einem qualitätsorientierten System muss notwendigerweise im Zentrum stehen. Zur Definition und Implementierung eines Qualitätsmanagementsystems und zu dessen kontinuierlichen Verbesserung stehen die Hilfsmittel und Vorgehensweisen des TotalQuality-Managements, das EFQM-Business-Excellence-Modell, EN ISO, VQG sowie weitere Tools zur Auswahl. Das Treffen von Qualitätszielen, die Identifikation externer und interner Kunden und das Begreifen des QM als Führungsinstrument und wesentlicher Faktor zur Motivation der Mitarbeiter sind sicherzustellen. «Qualität $=$ wenn der Kunde zurückkommt, nicht das Produkt.»

\section{Gesellschaftliche Trends 2015}

Niederländer, die auch im Krankheitsfall noch abenteuerlustig sind, können inzwischen zur Operation nach Südostasien reisen - und zwar auf Kosten ihrer Krankenversicherung. Bekannt-
Un nouvel absolu: art médical

\section{et gestion d'entreprise}

\section{Calculer, restructurer, optimiser, (se) perfectionner}

Les prestations de santé revêtent une grande importance, eu égard aux formes innovatrices des soins, au tassement des dépenses et aux taux de progression, malgré tout continuels, des dépenses de santé sur fond de société à quatre générations. La mutation du secteur de la santé place les médecins dans une position particulièrement exposée. Les compétences médicales acquises et les spécialisations qui s'ensuivent ne peuvent souvent plus suffire. Un changement de paradigme s'est produit dans ce secteur. Ce constat n'est ni récent, ni surprenant. Le plus étonnant serait toutefois l'absence ou le manque de disponibilité à reconnaître l'évidence des changements professionnels et à introduire dans le champ des connaissances médicales de nouveaux savoir-faire tels que la gestion d'unités, de services, d'institutions et de réseaux de la santé à tous les échelons et dans chaque fonction.

Cela dit, le chef d'un grand service de médecine ne sait en général pas ce que sa prestation coûte, ni ce qu'elle amène à son organisation. Comment doit-il planifier? Où peut-il optimiser les processus? Comment piloter les dépenses par patient, calculer les budgets ou réduire les coûts pour résister à la concurrence d'autres institutions? Des connaissances en gestion et un savoir-faire en économie d'entreprise sont une condition nécessaire à la survie professionnelle.

lich betragen dort die Behandlungsgebühren nur einen Bruchteil der europäischen und sollen das niederländische Gesundheitssystem entlasten. In der thailändischen Hauptstadt Bangkok hat inzwischen eine grosse Anzahl Kliniken eröffnet, die speziell auf ausländische OP-Touristen eingestellt sind. Die örtlichen Mediziner sind international ausgebildet, die Krankenschwestern vor Ort sind mehrsprachig und die Krankenzimmer sind ausnahmslos mit Satellitenfernsehen ausgestattet. Eines der übergeordneten Ziele Schweizer Business Schools kann es nur sein, Schweizer 
Spitalärzte und privatwirtschaftlich tätige Mediziner für Megatrends aus Wirtschaft, Politik und Gesellschaft zu sensibilisieren und sie zu befähigen, solche zu nutzen. «Probleme kann man niemals mit derselben Denkweise lösen, durch die sie entstanden sind.» (Albert Einstein)

\section{Rechtslehre und die Spezialitäten im Gesundheitswesen}

Mediziner müssen heute in der Lage sein, konkrete Sachverhalte im Bereich des Gesundheitswesens aus rechtlicher Sicht zu analysieren, Probleme $\mathrm{zu}$ erkennen und Lösungsansätze zu entwickeln. Eine solide Einführung in die juristische Denkweise ist hilfreich bei Themen wie Patientenrecht, Arzneimittelrecht, Gentechnologie, Datenschutz, Sozialversicherungsrecht und ähnlichem. «Das Fundament des Rechts ist die Humanität.» (Albert Schweitzer)

\section{Gesundheitsökonomie}

Ärztinnen und Ärzte erfassen die Grundlagen gesundheitsökonomischer Zusammenhänge mit Bezug auf das Gesundheitswesen als Teil der Volkswirtschaft. Gleichermassen gilt es, die Zusammenhänge zwischen ökonomischen und gesundheitsrelevanten Trends zu begreifen. Sie verstehen die Abhängigkeit von Gesundheitsleistungen und -produkten von den finanziellen Ressourcen und kennen die entsprechenden politischen Steuerungsinstrumente. «Wir müssen kapieren, nicht kopieren» (Manfred Perlitz)

\section{Projektmanagement, Zeitmanagement und Organisation}

Was ist effizientes Handeln? Effizientes Handeln ist im allgemeinen ziel- und planorientiert, realistisch, stabil-flexibel und Zeitverluste werden vermieden. Es ist besser, die richtige Arbeit zu tun (= Effektivität), als eine Arbeit nur «richtig» zu tun (= Effizienz). Neben dem Zeitmanagement muss ein Mediziner die Lebensphasen eines Projektes kennen und Kenntnis über die Funktionsmechanismen haben. Ein Spital als modern geführtes KMU kennt die Spannungsfelder: Kundenwünsche, Lieferanten, Partnermärkte, Wettbewerber, Unternehmen etc. Projekte in Spitälern und Kliniken scheitern häufig, weil sie nicht integrativ organisiert sind und/oder sich die verschiedenen Berufsgruppen einander nicht annähern (können). Ein Unternehmer im medizinalen Umfeld versteht Organisation als umfassende Managementaufgabe und ist mit dem entsprechenden Problemlösungsprozess vertraut. Er kennt die Elemente, Beziehungen und Dimensionen einer organisatorischen Regelung und die damit zusammenhängenden Methoden und
Techniken der Realisierung - und er kennt und bewertet die wichtigsten Organisationsformen aus der Praxis. Zudem analysiert er die Chancen und Risiken von Reorganisation. "Zwischen zu früh und zu spät liegt immer nur ein Augenblick.» (Franz Werfel)

\section{Wissensmanagement \& Personalführung}

Wie gehen wir mit der wichtigsten Ressource um, die unsere Volkswirtschaft hat, mit den Menschen? Warum sollte man sich um Veränderung bemühen, wenn scheinbar alles gut läuft? Selbst bei erfolgreichen Unternehmen oder gar Marktführern schlummert hier noch ein riesiges Potential. Ein Paradebeispiel ist das 1859 gegründete Mannheimer Unternehmen Boehringer \& Söhne. In seinem 145. Lebensjahr ist das Pharmaunternehmen bei bester Gesundheit, heisst inzwischen Roche Pharma und verkauft seine Produkte in mehr als 150 Länder dieser Welt. «Ein Spitzenplatz ist kein Rastplatz», so die Geschäftsleitung. In der heutigen Wissensgesellschaft ist der Mensch der Schlüssel für mehr Leistung. Das Management muss sich klar sein hinsichtlich der Bedeutung neuer Strukturen. Wie organisiere ich den Aufbruch und weiteren Fortgang meiner Belegschaft in die Wissensgesellschaft? Roche hat hierzu das Instrument «My Work Place» («Meine Arbeitswelt») eingeführt. Es dient der Selbstreflexion des einzelnen und macht zugleich die verborgenen Strukturen im Unternehmen, in der Abteilung und unter den Mitarbeitern sichtbar. Generell ist die gezielte Aus- und Weiterbildung der Mitarbeiter eine vordringliche Aufgabe jeder Personalabteilung und dennoch, es gibt kaum einen Bereich, in dem es in einem Unternehmen schlechter bestellt ist. Kostensenkung bei gleichzeitiger Steigerung der Effizienz scheint im Personalbereich nicht $\mathrm{zu}$ funktionieren. "Wissen = der einzige Produktionsfaktor, der nicht unter das Gesetz des abnehmbaren Ertrags fällt.» (John Maurice Clark)

\section{Risk Management}

Risiken sind kein Schicksal! Folgt man dem Entscheidungsmanagement, treffen wir eben diese entweder unter Unsicherheit oder eben unter Risiko. Die Logik eines Ignoranten ist simpel: Was ich nicht weiss, macht mich nicht heiss. Deshalb gehen viele Mitmenschen nicht zum Gesundheitscheck oder zur Krebsvorsorge. So ähnlich geschieht das häufig auch in Organisationen und Firmen, und dieses Verhalten zieht sich quer durch alle Branchen und Tätigkeitsbereiche: Was nicht leicht gemessen werden kann, wissen Unternehmen nicht. Deswegen kümmern sie sich nicht darum. Unternehmen heisst 
riskieren. Erfolgreich unternehmen heisst kalkuliert riskieren. «Die Ablehnung eines Risikos ist für ein Unternehmen das grösste Risiko.» (Reinhard Mohn)

\section{Arzt - Mediziner - Manager: Der Wandel der Zeit}

Die oben dargestellten betriebswirtschaftlichen Themenfelder sowie eine ganze Reihe weiterer, so etwa das Wissen um den Einsatz der richtigen Rhetorik, Vortrags- und Präsentationstechnik, solide Kenntnisse im Marketing seiner Organisation, wie erfolgskritisch Outsourcing-Entscheidungen in Spitälern sein können, wohin die Allianzen im Gesundheitswesen führen usw., gehören schon heute - hier und jetzt - zum Repertoire eines «Doktors». Die Mediziner im westlichen Teil Europas müssen vollkommen neue Wege gehen - eine gezielte, ganzheitliche betriebswirtschaftliche Weiterbildung an einer Business School gehört in der zweiten Hälfte des ersten Millenniumjahrzehnts zum Standard. Die Forderung, «Wir brauchen Ärzte, keine Manager», der deutschen Ärztekammern sind inzwischen ohne Bestand. Vor zehn Jahren hat noch niemand von Clustern oder Managed Care gesprochen. Heute sind Cluster (auch Medizinalcluster) ein Schlüssel für Wirtschaftswachstum in Europa. Sie stehen für Regionalentwicklung und damit für Arbeitsplätze, Netzwerke, Traditionen, für neue Technologien und für das Zusammenspiel von Wirtschaft und Wissenschaft. Die Verlagerung des Versicherungsrisikos von den Krankenversicherern zu den Spitälern hat stattgefunden. Inzwischen begreift jeder Mediziner den Einfluss von Elementen der Managed Care auf die diversen Anspruchsberechtigten im Gesundheitswesen. Angesichts der demographischen Entwicklung können wir uns die moderne Medizin wohl (bald) nicht mehr leisten. Es ist mathematischer Unsinn, zu glauben, dass jeder Bürger am medizinischen Fortschritt teilhaben kann. Genau deshalb werden die betriebswirtschaftlichen Kenntnisse der Mediziner gebraucht, um mit hoher Effizienz und Effektivität möglichst viele Klienten bedienen zu können.

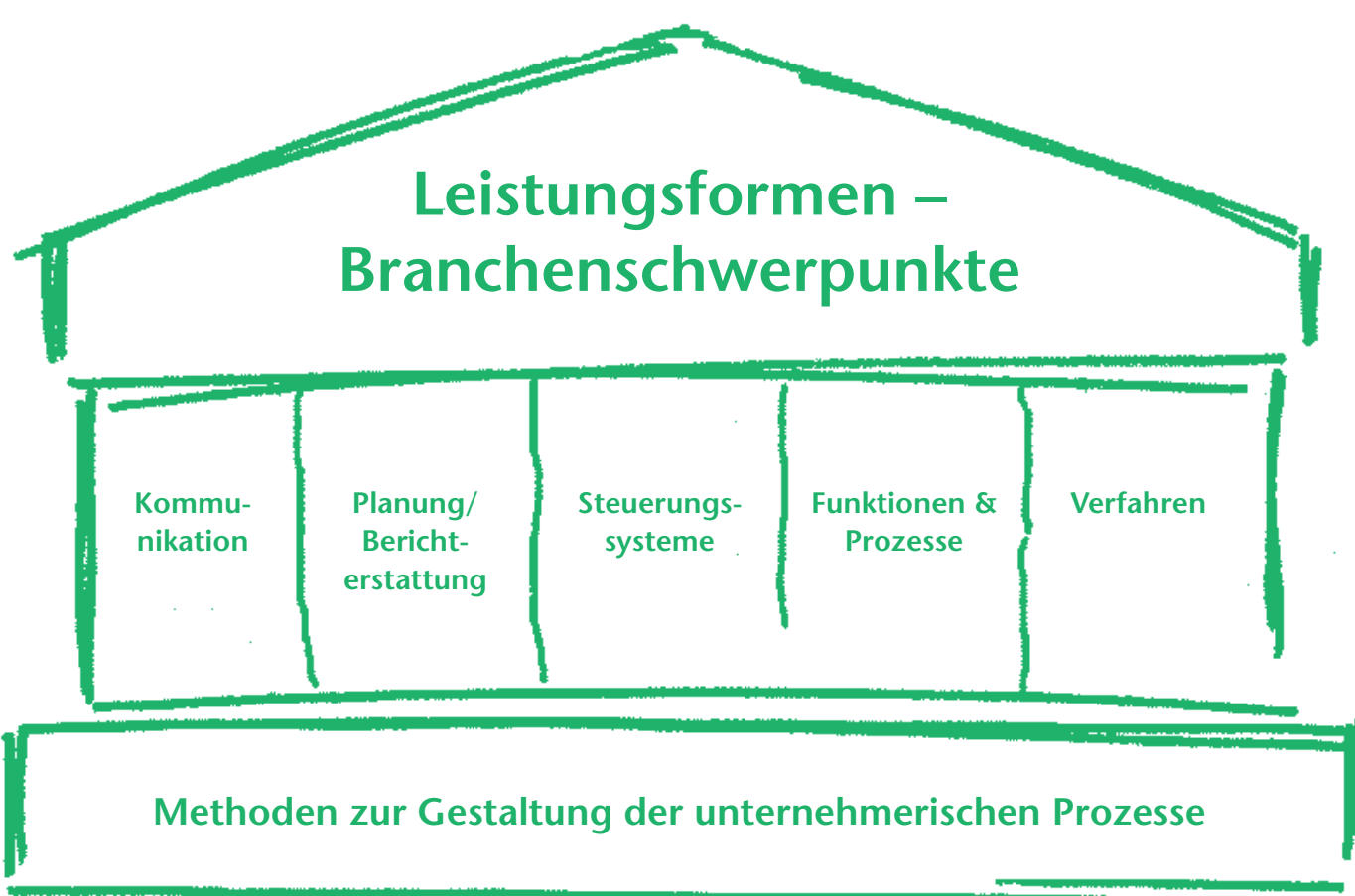

